\title{
Soluble Heparin-Binding EGF-Like Growth Factor (HB-EGF) is a Potential Serological Biomarker for Various Cancer Types
}

Noriyuki Kasai ${ }^{1 *}$, Yukitaka Yoshikawa ${ }^{1}$, Kazuhiro Kobayashi' ${ }^{1}$, Shinobu Shioya ${ }^{1}$, Shingo Miyamoto ${ }^{2}$, Eisuke Mekada ${ }^{3}$ and Junichi

Enokizono ${ }^{1}$

${ }^{1}$ Pharmacokinetic Research Laboratories, Kyowa Hakko Kirin Co. Ltd., Shimotogari, Nagaizumi-cho, Sunto-gun, Japan

${ }^{2}$ Department of Obstetrics and Gynecology, School of Medicine, Fukuoka University, 7-Chome Nanakuma, Jonan-ku, Fukuoka, Japan

${ }^{3}$ Research Institute for Microbial Diseases, Osaka University, Yamadaoka, Suita, Osaka, Japan

\begin{abstract}
Background: Heparin-binding EGF-like Growth Factor (HB-EGF), a member of the EGF family, exerts its biological activity through activation of the EGF receptors. HB-EGF is initially synthesized as a membrane-anchored precursor protein (proHB-EGF), and then proteolytically cleaved, resulting in the mitogenically active soluble form. HB-EGF plays pivotal roles in many physiologic and pathologic processes such as development and cell proliferation. In this study, we measured soluble HB-EGF concentrations in serum samples obtained from healthy volunteers and patients of various cancer types.
\end{abstract}

Materials and methods: Soluble HB-EGF levels in human serum samples were quantified by the immuno-PCR method.

Results: The mean soluble HB-EGF levels of the 20 healthy volunteers and 10 colon, breast, ovarian, head and neck, non-small cell lung, pancreatic, and small cell lung cancer patients were 5.04, 18.0, 15.1, 10.4, 6.69, 9.78, 23.6, and $5.60 \mathrm{pg} / \mathrm{mL}$, respectively. There was a statistically significant difference between the HB-EGF concentrations of healthy volunteers and patients in 5 out of 7 cancer types. Furthermore, a trend for HB-EGF levels to increase along with disease stage was observed.

Conclusion: Soluble HB-EGF may be a useful diagnostic serological biomarker for various cancer types, and a predictive and/or pharmacodynamic biomarker for HB-EGF-targeted therapeutics.

Keywords: HB-EGF; Cancer; Serological biomarker; Immuno-PCR

Abbreviations: HB-EGF: Heparin-Binding EGF-like growth factor; EGF: Epidermal Growth Factor; LLOQ: Lower Limit Of Quantification

\section{Introduction}

Epidermal Growth Factor (EGF) receptors and EGF family members are well-known as promising targets for cancer therapeutics. Heparin-binding EGF-like Growth Factor (HB-EGF), a member of the EGF family, is also a notable therapeutic target in several types of human cancers. HB-EGF exerts its biological activity through activation of HER1 and HER4 [1-3], and plays a pivotal role in many physiologic and pathologic processes via transduction of extracellular signals [4-6].

In common with other EGF family members, HB-EGF is initially synthesized as a membrane-anchored precursor protein (proHBEGF). The transmembrane form of HB-EGF is comprised of a signal peptide, propeptide, heparin-binding, EGF-like, juxta membrane, transmembrane, and cytoplasmic domains [1]. Subsequently, proHBEGF is cleaved at its juxta membrane domain by metalloproteases in a process known as ectodomain shedding [7], yielding a soluble form of HB-EGF, which is a potent mitogen and chemo-attractant for a number of cell types expressing its relevant receptors [4].

In our previous study, we developed an immuno-PCR method for the determination of soluble HB-EGF concentrations in human serum [8]. High specificity and sensitivity of the assay enabled us to demonstrate that ovarian cancer patients had significantly higher levels of HB-EGF than healthy volunteers [8]. The results appear to be consistent with many reports indicating the importance of HB-EGF in ovarian cancer [9-11].

In this study, we measured soluble HB-EGF concentrations in serum samples obtained from healthy volunteers and patients of various cancer types in order to examine the possibility of soluble HB-EGF as a useful diagnostic serological biomarker for multiple cancer types.

\section{Materials and Methods}

\section{Materials}

A goat polyclonal anti-HB-EGF antibody and recombinant human HB-EGF were purchased from R\&D systems (Minneapolis, MN, USA). A mouse monoclonal anti-HB-EGF antibody, KM3566 (Patent Application Publication No. US 2011/0052595 A1) was obtained from Kyowa Hakko Kirin (Tokyo, Japan).

\section{Matrix}

Serum samples of healthy volunteers were purchased from Uniglobe Research Corporation (Reseda, CA, USA). Patient serum samples of colon, breast, ovarian, head and neck, non-small cell lung, small cell lung, and pancreatic cancer were purchased from Bioreclamation (Hicksville, NY, USA).

Measurement of soluble HB-EGF levels in human serum by immuno-PCR method

Soluble HB-EGF levels in human serum samples were quantified by the previously established assay method [8].

*Corresponding author: Noriyuki Kasai, Pharmacokinetic Research Laboratories, Kyowa Hakko Kirin Co. Ltd., 1188, Shimotogari, Nagaizumi-cho, Sunto-gun, Shizuoka, 411-8731, Japan, Tel: +81-55-989-4289; Fax: +81-55-989-2073; E-mail: noriyuki.kasai@kyowa-kirin.co.jp

Received December 09, 2013; Accepted December 20, 2013; Published December 23, 2013

Citation: Kasai N, Yoshikawa Y, Kobayashi K, Shioya S, Miyamoto S, et al (2013) Soluble Heparin-Binding EGF-Like Growth Factor (HB-EGF) is a Potential Serological Biomarker for Various Cancer Types. J Mol Biomark Diagn 5: 158 doi:10.4172/2155-9929.1000158

Copyright: ( 2013 Kasai N, et al. This is an open-access article distributed under the terms of the Creative Commons Attribution License, which permits unrestricted use, distribution, and reproduction in any medium, provided the original author and source are credited 
Citation: Kasai N, Yoshikawa Y, Kobayashi K, Shioya S, Miyamoto S, et al. (2013) Soluble Heparin-Binding EGF-Like Growth Factor (HB-EGF) is a Potential Serological Biomarker for Various Cancer Types. J Mol Biomark Diagn 5: 158. doi:10.4172/2155-9929.1000158

\section{Other methods}

Statistical analysis was conducted using SAS ${ }^{\bullet}$ (Release 9.2, SAS Institute, Cary, NC, USA).

\section{Results}

\section{Measurement of soluble HB-EGF levels in human serum by immuno-PCR method}

Soluble HB-EGF levels in human serum samples were quantified by the immuno-PCR system. Serum samples with concentrations of lower than the LLOQ were regarded as the LLOQ, $5 \mathrm{pg} / \mathrm{mL}$. Figure 1 shows a plot of HB-EGF concentrations in serum samples of healthy volunteers $(n=20)$ and various cancer patients $(n=10$ each). The mean soluble HB-

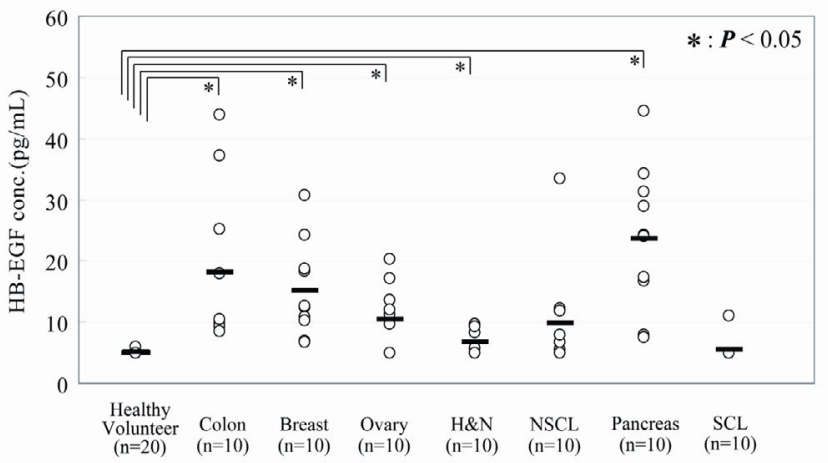

Figure 1: Comparison of serum HB-EGF concentrations determined by immuno-PCR method between healthy volunteers $(n=20)$ and patients of various types of cancers ( $n=10$ of each).

The lower limit of quantification was $5 \mathrm{pg} / \mathrm{mL}$, and serum HB-EGF concentrations below the limit of quantification were regarded as $5 \mathrm{pg} / \mathrm{mL}$. Open circles represent the individual HB-EGF concentrations. Bars indicate mean. The results of $t$ test suggested that there was a statistically significan difference between the values of healthy volunteers and patients of colon, breast, ovarian, head and neck, and pancreatic cancers $(P<0.05$, Welch's test). (H\&N: Head and neck, NSCL: Non-small cell lung, SCL: Small cell lung)

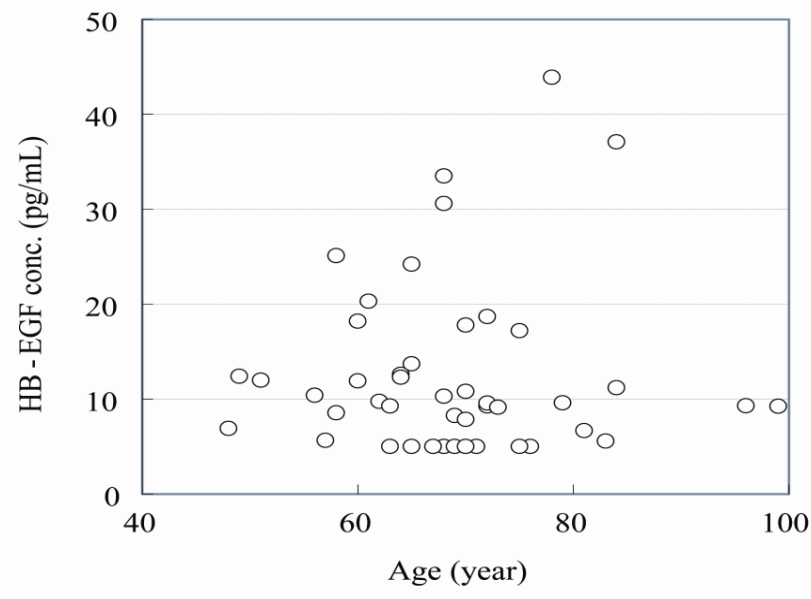

Figure 2: Individual plots of ages and serum HB-EGF concentrations determined by immuno-PCR method.

The lower limit of quantification was $5 \mathrm{pg} / \mathrm{mL}$, and serum HB-EGF concentrations below the limit of quantification were regarded as $5 \mathrm{pg} / \mathrm{mL}$. Open circles represent the individual HB-EGF concentrations $(n=69)^{a}$ ). There was no correlation between ages and HB-EGF concentrations. ${ }^{\text {a) }}$ One out of 70 patients had no information regarding disease stage.
EGF concentrations of the 20 healthy volunteers and 10 colon, breast, ovarian, head and neck, non-small cell lung, pancreatic, and small cell lung cancer patients were 5.04,18.0,15.1, 10.4, 6.69, 9.78, 23.6, and $5.60 \mathrm{pg} / \mathrm{mL}$, respectively. The individual soluble HB-EGF levels in the patient serum samples examined are shown in Supplementary data with the attachment of clinical characteristics provided by the vendor of the serum samples. The statistic analyses by Welch's $t$ test demonstrated a statistically significant difference between the HB-EGF concentrations of healthy volunteers and patients in 5 out of 7 various cancer types, i.e., colon, breast, ovarian, head and neck, and pancreatic cancer; $\mathrm{P}<0.05$.

\section{Discussion}

In this study, we determined soluble HB-EGF concentrations in human serum by the immuno-PCR method. As a result, there was a statistically significant difference between the HB-EGF concentrations of healthy volunteers and patients in 5 out of 7 various cancer types, i.e., colon, breast, ovarian, head and neck, and pancreatic cancer. In our previous study, we demonstrated that ovarian cancer patients had significantly higher levels of soluble HB-EGF than healthy volunteers [8]. The results of our current study in which a different set of ovarian and other cancer patient samples were used reaffirmed the potential of serum HB-EGF as a biomarker in ovarian cancer and suggested the possible application to other cancer types.

There are several academic publications supporting our results, as follows. HB-EGF is broadly expressed in human carcinomas such as pancreatic and colon cancers [12], and thus HB-EGF-targeted cancer therapy is under clinical trial [13]. HB-EGF mRNA is reported to increase in human pancreatic cancer as compared to normal pancreas, suggesting an important role of HB-EGF in pancreatic cancer cell growth [14]. Ito et al. reported the positive expression of HB-EGF protein in approximately $70 \%$ of breast carcinoma cases and negative or faint expression in normal mammary glands [15]. Their group also demonstrated the positive expression of HB-EGF protein in pancreatic adenocarcinoma [16]. Furthermore, another group suggested a crucial role of HB-EGF in the aggressive behavior of head and neck squamous cell carcinoma [17]. In addition, there are many reports indicating the importance of HB-EGF in ovarian cancer. HB-EGF, especially its soluble form, was reported to be essential for tumor growth of ovarian cancer cells in nude mice [9]. Clinically, HB-EGF concentrations in peritoneal fluid of ovarian cancer patients were shown to be significantly higher than those in ovarian cyst patients or normal ovary patients [10]. It has also been shown that HB-EGF expression is significantly associated with a negative clinical outcome in ovarian cancer [11].

Exploring statistical correlation analysis between serum HB-EGF levels and clinical data (e.g. history of medications, disease stage, and current condition) shouldbe of immediateinterest. Theplots of individual soluble HB-EGF levels of the patient serum samples demonstrated that there is no correlation between serum HB-EGF concentrations and age (Figure 2), whereas HB-EGF levels demonstrated a trend to increase along with disease stage (Figure 3). However, it should be noted that the disease stages of patients analyzed in this study are one-sided in some cancer types (e.g. colon cancer; mostly stage III/IV). Thus, further analysis with more data will be required to demonstrate the statistically meaningful correlation between serum HB-EGF concentrations and disease stages within each cancer type.

Serum HB-EGF detected by our method is a possible early diagnostic marker for several cancer types since it was detected even in early disease stages (i.e. stage I/II). Furthermore, combinations of soluble HB-EGF and well-established serological biomarkers such as 
Citation: Kasai N, Yoshikawa Y, Kobayashi K, Shioya S, Miyamoto S, et al. (2013) Soluble Heparin-Binding EGF-Like Growth Factor (HB-EGF) is a Potential Serological Biomarker for Various Cancer Types. J Mol Biomark Diagn 5: 158. doi:10.4172/2155-9929.1000158

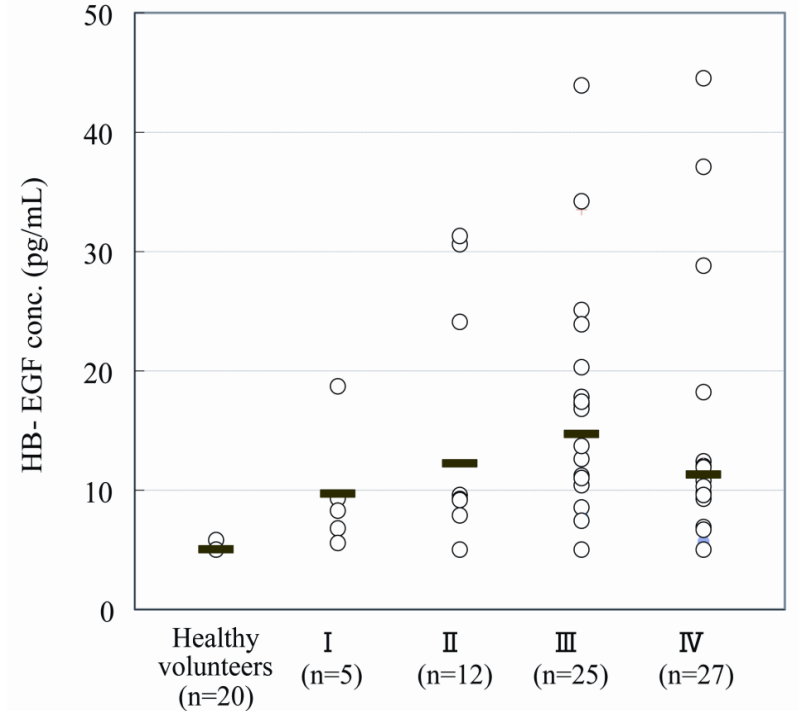

Figure 3: Individual plots of disease stages and serum HB-EGF concentrations determined by immuno-PCR method.The lower limit of quantification was 5 $\mathrm{pg} / \mathrm{mL}$, and serum HB-EGF concentrations below the limit of quantification were regarded as $5 \mathrm{pg} / \mathrm{mL}$. Open circles represent the individual HB-EGF concentrations $(n=69)^{a}$. Bars indicate mean. This plot shows a tendency that higher serum HB-EGF concentrations are observed in higher stages. ${ }^{\text {a) }}$ One out of 70 patients had no information regarding disease stage.

serum CA125 for ovarian cancer [18] and CA19-9 for pancreatic cancer [19] may increase the accuracy of screening, diagnosis, prediction of prognosis, etc.

In summary, we demonstrated a statistically significant difference between the HB-EGF concentrations of healthy volunteers and patients in various cancer types. Furthermore, a trend for HB-EGF levels to increase along with disease stage was observed. These results suggest that soluble HB-EGF may be a useful diagnostic serological biomarker for various cancer types, and a predictive and/or pharmacodynamic biomarker for HB-EGF-targeted therapeutics.

\section{Acknowledgement}

We thank Drs. H. Ishida, Y. Ohki, T. Tawara, V. Strout, and H. Kaito for excellent support and helpful suggestion.

\section{Appendix-Supplementary data}

Clinical characteristics of cancer patients examined (i.e., age, gender, medication, and disease stage) and HB-EGF concentrations determined by immuno-PCR method were listed in Supplementary data.

\section{References}

1. Higashiyama S, Lau K, Besner GE, Abraham JA, Klagsbrun M (1992) Structure of heparin-binding EGF-like growth factor. Multiple forms, primary structure, and glycosylation of the mature protein. J Biol Chem 267: 6205-6212.

2. Higashiyama S, Abraham JA, Miller J, Fiddes JC, Klagsbrun M (1991) A heparin-binding growth factor secreted by macrophage-like cells that is related to EGF. Science 251: 936-939.

3. Elenius K, Paul S, Allison G, Sun J, Klagsbrun M (1997) Activation of HER4 by heparin-binding EGF-like growth factor stimulates chemotaxis but not proliferation. EMBO J 16: 1268-1278.
4. Raab G, Klagsbrun M (1997) Heparin-binding EGF-like growth factor. Biochim Biophys Acta 1333: F179-199.

5. Harris RC, Chung E, Coffey RJ (2003) EGF receptor ligands. Exp Cell Res 284: $2-13$

6. Higashiyama S, Nanba D (2005) ADAM-mediated ectodomain shedding of HBEGF in receptor cross-talk. Biochim Biophys Acta 1751: 110-117.

7. Goishi K, Higashiyama S, Klagsbrun M, Nakano N, Umata T, et al. (1995) Phorbol ester induces the rapid processing of cell surface heparin-binding EGF-like growth factor: conversion from juxtacrine to paracrine growth factor activity. Mol Biol Cell 6: 967-980.

8. Kasai N, Kobayashi K, Shioya S, Yoshikawa Y, Yotsumoto F, et al. (2012) Soluble heparin-binding EGF-like growth factor (HB-EGF) detected by newly developed immuno-PCR method is a clear-cut serological biomarker for ovarian cancer. Am J Transl Res 4: 415-421.

9. Miyamoto S, Hirata M, Yamazaki A, Kageyama T, Hasuwa H, et al. (2004) Heparin-binding EGF-like growth factor is a promising target for ovarian cance therapy. Cancer Res 64: 5720-5727.

10. Yagi H, Miyamoto S, Tanaka Y, Sonoda K, Kobayashi H, et al. (2005) Clinical significance of heparin-binding epidermal growth factor-like growth factor in peritoneal fluid of ovarian cancer. Br J Cancer 92: 1737-1745

11. Tanaka Y, Miyamoto S, Suzuki SO, Oki E, Yagi H, et al. (2005) Clinica significance of heparin-binding epidermal growth factor-like growth factor and a disintegrin and metalloprotease 17 expression in human ovarian cancer. Clin Cancer Res 11: 4783-4792.

12. Miyamoto S, Yagi H, Yotsumoto F, Kawarabayashi T, Mekada E (2006) Heparinbinding epidermal growth factor-like growth factor as a novel targeting molecule for cancer therapy. Cancer Sci 97: 341-347.

13. Yagi $H$, Yotsumoto F, Sonoda K, Kuroki M, Mekada E, et al. (2009) Synergistic anti-tumor effect of paclitaxel with CRM197, an inhibitor of HB-EGF, in ovarian cancer. Int J Cancer 124: 1429-1439.

14. Kobrin MS, Funatomi H, Friess H, Buchler MW, Stathis $P$, et al. (1994) Induction and expression of heparin-binding EGF-like growth factor in human pancreatic cancer. Biochem Biophys Res Commun 202: 1705-1709.

15. Ito Y, Takeda T, Higashiyama S, Noguchi S, Matsuura N (2001) Expression of heparin-binding epidermal growth factor-like growth factor in breast carcinoma. Breast Cancer Res Treat 67: 81-85.

16. Ito Y, Takeda T, Higashiyama S, Takeda T, Yamamoto Y, et al. (2001) Expression of Heparin-Binding Epidermal Growth Factor-like Growth Factor in Pancreatic Adenocarcinoma. Int J Pancreatol 29: 47-52.

17. Ohnishi Y, Inoue H, Furukawa M, Kakudo K, Nozaki M (2012) Heparin-binding epidermal growth factor-like growth factor is a potent regulator of invasion activity in oral squamous cell carcinoma. Oncol Rep 27: 954-958.

18. Kobayashi E, Ueda Y, Matsuzaki S, Yokoyama T, Kimura T, et al. (2012) Biomarkers for screening, diagnosis, and monitoring of ovarian cancer. Cancer Epidemiol Biomarkers Prev 21: 1902-1912.

19. Winter JM, Yeo CJ, Brody JR (2013) Diagnostic, prognostic, and predictive biomarkers in pancreatic cancer. J Surg Oncol 107: 15-22. 